\title{
Circulation and formation number of laminar vortex rings
}

\author{
By MOSHE ROSENFELD ${ }^{1}$, EDMOND RAMBOD \\ AND MORTEZA GHARIB ${ }^{2}$ \\ ${ }^{1}$ Faculty of Engineering, Tel Aviv University, Tel Aviv 69978, Israel \\ ${ }^{2}$ Graduate Aeronautical Laboratories, California Institute of Technology, Pasadena, CA, USA
}

(Received 27 January 1997 and in revised form 24 July 1998)

The formation time scale of axisymmetric vortex rings is studied numerically for relatively long discharge times. Experimental findings on the existence and universality of a formation time scale, referred to as the 'formation number', are confirmed. The formation number is indicative of the time at which a vortex ring acquires its maximal circulation. For vortex rings generated by impulsive motion of a piston, the formation number was found to be approximately four, in very good agreement with experimental results. Numerical extensions of the experimental study to other cases, including cases with thick shear layers, show that the scaled circulation of the pinched-off vortex is relatively insensitive to the details of the formation process, such as the velocity programme, velocity profile, vortex generator geometry and the Reynolds number. This finding might also indicate that the properly scaled circulation of steady vortex rings varies very little. The formation number does depend on the velocity profile. Non-impulsive velocity programmes slightly increase the formation number, while non-uniform velocity profiles may decrease it significantly. In the case of a parabolic velocity profile of the discharged flow, for example, the formation number decreases by a factor as large as four. These findings indicate that a major source of the experimentally found small variations in the formation number is the different evolution of the velocity profile of the discharged flow.

\section{Introduction}

Vortex rings have attracted considerable interest for a long time. Not only do they exhibit spectacular flow fields but they are of major importance in both fundamental and practical flow problems. Naturally, a great volume of research has been published on vortex rings; for recent reviews see Shariff \& Leonard (1992) and Lim \& Nickels (1995). The mathematical foundations are given in Saffman (1992).

A new feature of vortex ring formation was recently observed experimentally by Gharib, Rambod \& Shariff (1998, henceforth denoted as GRS) for vortex rings formed by a piston pushing a column of fluid out of a tube. As the duration of the piston stroke (or discharge time) increased, GRS showed evidence for the existence of a limiting process that imposes an upper bound on the circulation a vortex ring can acquire for a given set of flow parameters. They suggested that this limiting process is the outcome of the Kelvin-Benjamin variational principle for steady axistouching vortex rings. This variational principle states that the kinetic energy of impulse-preserving rearrangements of the vorticity field by an arbitrary divergencefree velocity field is maximum for a steady vortex ring. This maximum of the vortex 
circulation was found in the experiments to be produced by the piston at a narrow range of formation time $4.5>t^{*}>3.6$ for a number of cases differing in the velocity programme and in the velocity or diameter of the piston. This universal number, that may define a formation time scale of vortex rings, is referred to as the formation number. Any further discharge of fluid does not increase the vortex ring circulation. Instead, the excess vorticity is accumulated in the wake (tail) of the vortex.

Following GRS, the formation time $t^{*}$ is defined by

$$
t^{*}=\frac{\bar{U}_{p} t}{D},
$$

where $\bar{U}_{p}$ is the mean velocity discharged from a tube of diameter $D$ and $t$ is the time ( $\bar{U}_{p}$ at the stop of the piston is used). In many existing experimental works, vortex rings are generated by pushing a column of fluid of length $L(t)$ out of a nozzle or an orifice using a piston. Analytical works refer to this situation using the slug flow model, e.g. Glezer (1988) or Shariff \& Leonard (1992). In these cases the formation time is given by $L(t) / D=\bar{U}_{p} t / D$. The formation time when the discharge of fluid stops (i.e. at the stop of the pushing of the piston) is thus given by $T^{*}=L_{m} / D$, where $L_{m}$ is the maximal stroke. The two equivalent terms $T^{*}$ and $L_{m} / D$ are used therefore interchangeably throughout this study.

The existence of a universal formation number is intriguing, as GRS themselves mentioned. It hints at the possibility of nature using this time scale for certain evolutionary incentives, such as optimum ejection of blood into the left ventricle or aquatic locomotion processes where ejection of vortices might have been utilized for propulsion.

In the present paper we seek, using computational fluid dynamics techniques, to validate, complement and extend the experimental findings of GRS by investigating the laminar vortex ring formation process and the factors influencing the evolution of the total and vortex circulations as well as the formation number. In particular, we study numerically flow cases that are difficult or even impossible to investigate experimentally. Such is the case in the study of the dependence of the vortex ring's circulation on the velocity profile of the discharging jet. In the experimental set-up, the velocity profile is determined by the motion of the piston and the geometry of the orifice or the nozzle, Didden (1979). The streamwise velocity component is neither uniform nor parabolic; rather it evolves in time depending on the velocity programme, piston stroke ratio and Reynolds number. However, in studying the effect of the discharge velocity distribution on the circulation of the vortex ring, for example, it is useful to impose known velocity profiles. This task is next to impossible in experimental studies but is very easy to perform in numerical simulations.

The present paper is a continuation of the experimental study of GRS. Therefore, the flow processes involved, as well as existing analytical and experimental works are not repeated here. Rather, we focus on reviewing related numerical studies of laminar flow cases only. The number of numerical studies of vortex rings using the Navier-Stokes equations is quite small. Stanaway, Cantwell \& Spalart (1988) employed a specially designed spectral method for simulating a single vortex ring over a wide range of Reynolds numbers as well as the leapfrogging of two vortex rings. Shedding of vorticity from the ring into the wake was found. Orlandi \& Verzicco (1993) simulated the impingement process of vortex rings on flat boundaries while Weidman \& Riley (1993) considered two concentric vortex rings. The two latter studies employed finite difference schemes with a resolution of $O\left(10^{4}\right)$ mesh points and Reynolds numbers of $O\left(10^{3}\right)$ based on the initial circulation of the vortex ring. 
However, none of these studies considered the formation process of the vortex rings; rather, they started from an assumed vorticity distribution. The focus of the present study is on the formation process and therefore these works cannot shed light on the topics of the present study and in particular on the formation number of vortex rings.

The numerical works of Nitsche \& Kransy (1994) and James \& Madnia (1996) are of direct relevance because they did simulate the formation of vortex rings. Nitsche \& Kransy (1994) reproduced Didden's (1979) experiments (that considered a small stroke ratio of $\left.L_{m} / D<2.6\right)$ using a vortex sheet model and $L_{m} / D<1.5$. Good global agreement with the experiments was found, although the details depend on an arbitrarily chosen artificial smoothing parameter. Obviously, the vortex sheet model has certain disadvantages and the full Navier-Stokes equations are required for calculating the formation phase of relatively low Reynolds number flows. James \& Madnia (1996) indeed used the Navier-Stokes equations for simulating the formation and propagation of a single vortex ring for different velocity programs and for both orifice and nozzle vortex generators (the latter case was simulated using second-order derivative boundary conditions on the upstream boundary). Comparisons with the experimental results of Didden (1979) were presented as well and the circulation, impulse and in some cases the vorticity field were depicted for five cases differing in the velocity programme and geometry configuration (nozzle or orifice). They employed the compressible Navier-Stokes equations for a relatively low Mach number of 0.4 (based on the maximal value of the discharge velocity). The compressibility effects were not assessed, although they were not anticipated to modify the conclusions of their study significantly, at least not the qualitative description of the flow. The results of James \& Madnia (1996) too are limited to small stroke ratios $\left(L_{m} / D<4\right)$. In the present study we are interested in larger stroke ratios as well as in the relationship between the evolution of the circulation and the formation time scale of laminar vortex rings.

In $\S 2$ the numerical model and method are briefly described and mesh and time step refinement studies are elaborated. In $\S 3$, the basic features of the vortex ring formation are presented as a function of $T^{*}$ (or $L_{m} / D$ ) and comparisons with experimental results are performed. In that section, as well as in the remaining sections, the main focus is on the evolution of the total and vortex circulations. In $\S \S 4$ and 5 we discuss the dependence of the vortex circulation and formation number on the velocity programme, velocity profile, Reynolds number and vortex generator configuration. It is shown that the scaled vortex circulation for the calculated cases is essentially invariant, and therefore the value of the formation number depends on the rate of total circulation generation.

\section{The numerical model}

The axisymmetric time-dependent incompressible Navier-Stokes equations in dimensionless form are employed for simulating the flow:

$$
\left.\begin{array}{c}
\frac{\partial u_{r}}{\partial t}+u_{r} \frac{\partial u_{r}}{\partial r}+u_{z} \frac{\partial u_{r}}{\partial z}=\frac{1}{r} \frac{\partial}{\partial r}\left(r \sigma_{r r}\right)-\frac{1}{r} \sigma_{\theta \theta}+\frac{\partial \sigma_{r z}}{\partial z}, \\
+u_{r} \frac{\partial u_{z}}{\partial r}+u_{z} \frac{\partial u_{z}}{\partial z}=\frac{1}{r} \frac{\partial}{\partial r}\left(r \sigma_{r z}\right)+\frac{\partial \sigma_{z z}}{\partial z}, \frac{1}{r} \frac{\partial}{\partial r}\left(r u_{r}\right)+\frac{\partial u_{z}}{\partial z}=0 .
\end{array}\right\}
$$




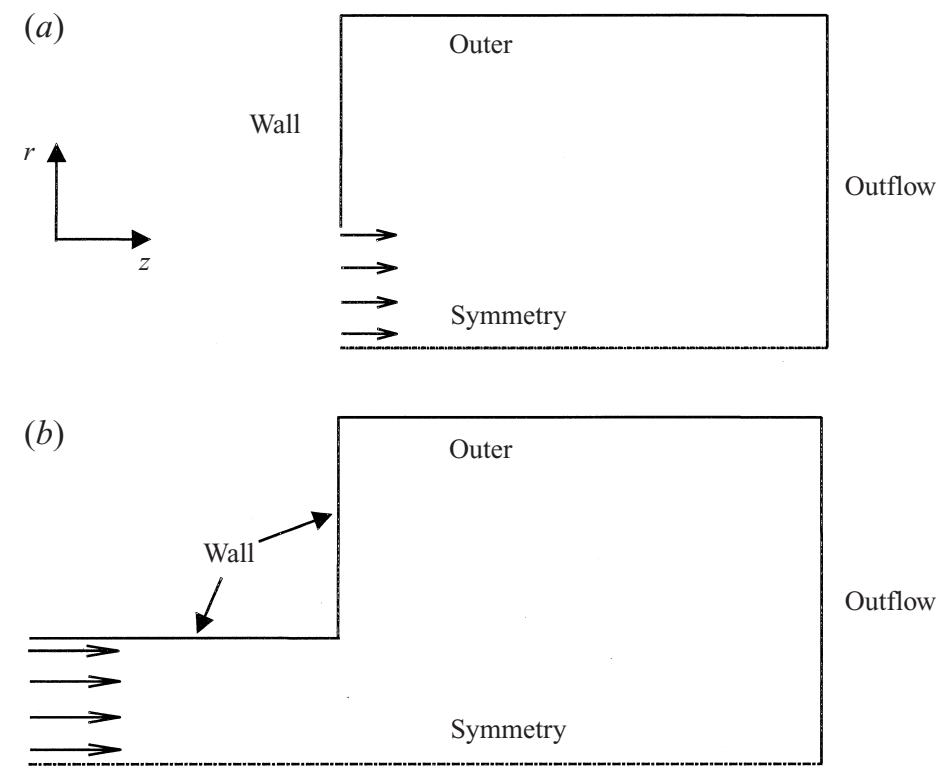

(c)

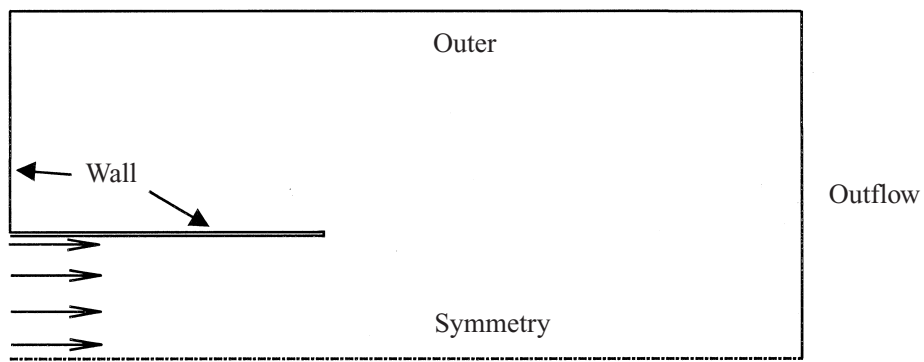

FiguRE 1. The domain of computations (not to scale) for: (a) specified discharge velocity (SDV), $(b)$ orifice and $(c)$ nozzle cases.

The stress components are given by

$$
\left.\begin{array}{ll}
\sigma_{r r}=-P+\frac{2}{\operatorname{Re}} \frac{\partial u_{r}}{\partial r}, & \sigma_{\theta \theta}=\frac{2}{\operatorname{Re}} \frac{u_{r}}{r}, \\
\sigma_{r z}=\frac{1}{R e}\left(\frac{\partial u_{r}}{\partial z}+\frac{\partial u_{z}}{\partial r}\right), & \sigma_{z z}=-P+\frac{2}{R e} \frac{\partial u_{z}}{\partial z} .
\end{array}\right\}
$$

The velocity components in the axial $(x)$ and radial $(r)$ directions are $u_{x}$ and $u_{r}$, respectively; $P$ is the non-dimensional pressure and $R e$ is the Reynolds number.

The computational domain used in most of the simulations is shown in figure $1(a)$. In this case the discharge velocity at the orifice-type generator is specified and therefore the flow inside the tube is not calculated. The orifice has a diameter of $D=2.5 \mathrm{~cm}$ and the outer boundary is placed at a distance of $H=5$ or $10 \mathrm{~cm}$ (which were found to be far enough not to affect the flow in the regions of interest). The downstream boundary was placed at a minimal distance of $40 \mathrm{~cm}$ and up to $120 \mathrm{~cm}$ (depending on the discharge velocity profile and the discharge time).

In a few other simulations the pushing of a column of fluid by the piston is modelled by specifying a uniform (time-dependent) velocity at the entrance to a tube 


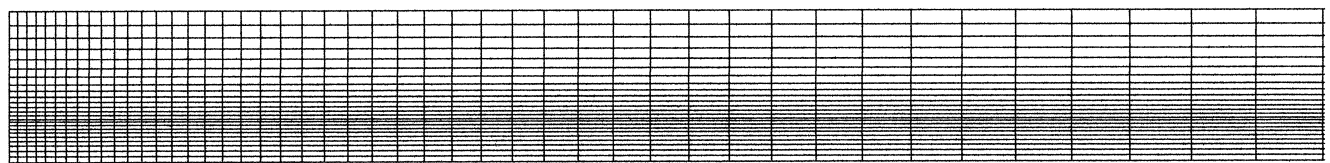

FIGURE 2. A sample mesh for the SDV case (every 5th mesh point is shown in each direction).

as depicted in figures $1(b)$ and $1(c)$. The magnitude of the imposed velocity is equal to the instantaneous piston velocity. Orifice (figure $1 b$ ) and nozzle (figure 1c) generator configurations were simulated by appropriately defining the computational zone and the boundary conditions. In these two latter cases, the velocity of the flow discharged from the tube develops in time similarly to the evolution of the discharge velocity profile in the experiments. We shall refer to the three different cases depicted in figure 1 as the specified discharge velocity (SDV), the orifice and the nozzle cases, respectively.

A zero initial velocity field was specified for all the cases. On the walls, the velocity was set to zero. On the outer boundary entrainment is allowed and on the outflow boundaries stress-free conditions are imposed. On the axis of symmetry, symmetry conditions are specified. In the SDV (specified discharge velocity) case, a uniform discharge velocity of $U_{p}=30 \mathrm{~cm} \mathrm{~s}^{-1}$ was imposed in most of the cases. In other runs, a Poiseuille flow is specified, maintaining the same mass flow rate (piston velocity). In the orifice or nozzle cases, a uniform velocity of $U_{p}=30 \mathrm{~cm} \mathrm{~s}^{-1}$ was specified at the upstream boundary of the tube. In most of the cases, an impulse velocity programme was used for a duration of $T \mathrm{~s}$ (that is equivalent to the maximal stroke $L_{m}$ of the piston in the experiments of GRS). In other cases a linear ramp or trapezoidal velocity programmes were specified for a finite duration with a maximal velocity of $U_{p}=30 \mathrm{~cm} \mathrm{~s}^{-1}$.

Time is scaled using (1.1), i.e. we shall refer to the formation time $t^{*}$. The Reynolds number in most of the calculations is $R e=2500$ (based on the maximal piston velocity $U_{p}$ and the tube or orifice diameter $D$ ).

\subsection{Discretization and numerical solution}

The axisymmetric Navier-Stokes equations (2) are solved by the commercially available finite element FIDAP package (Fluid Dynamics International, Evanston, IL). The implicit scheme is first-order accurate in time and second-order accurate in space; no numerical diffusion has been used. A projection formulation was selected with a segregated solver. Each linear system of algebraic equations was solved by a GMRES iterative solver. The convergence criterion of the relative change in the solution and in the residual was $0.1 \%$.

The standard mesh of the SDV case consists of $151 \times 121$ nodes in the axial and radial directions, respectively, and approximately 18000 quadrilateral linear four node elements. Node points were clustered in the vortex region as well as in the formation region. The adequate size of the mesh is determined by a mesh refinement study (see $\S 2.2$ ). A sample mesh for the SDV case is shown in figure 2 (for clarity only every fifth mesh point is shown in each direction). In the orifice and nozzle generator cases, the mesh downstream of the exit plane is identical to that of the specified velocity cases. Mesh points of similar density were added in the tube and outside it (in the nozzle case). 


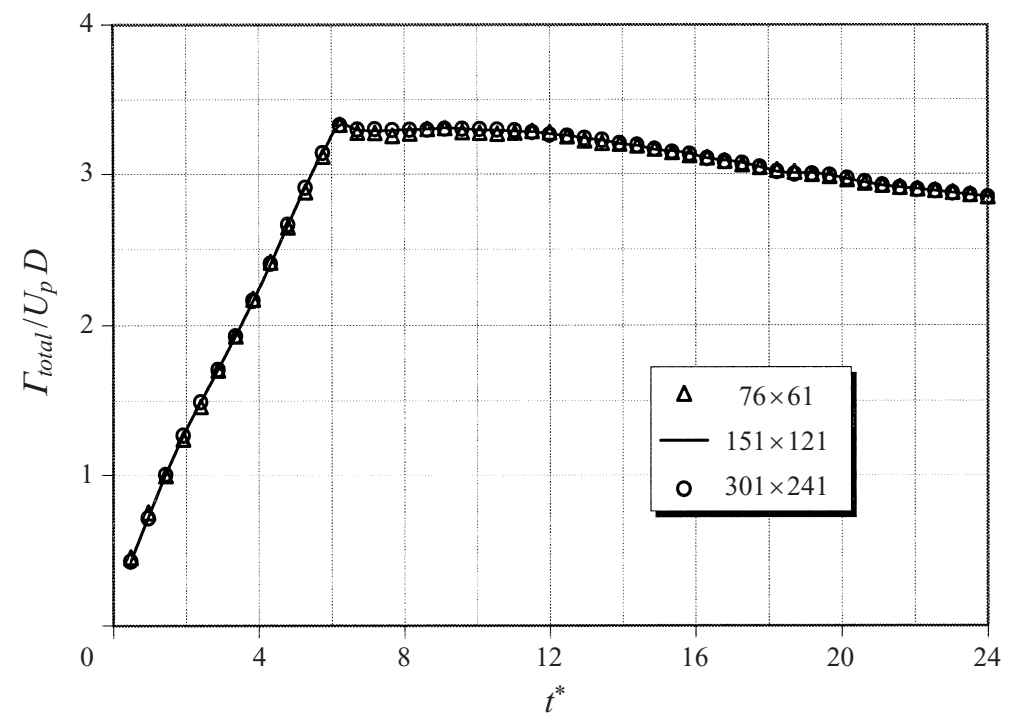

FIGURE 3. Mesh refinement study of the total circulation $\left(\Gamma_{\text {total }}\right)$.

\subsection{Validation}

The computational results are validated by performing mesh and time-step refinement studies as well as by comparing with experimental results. In the present section, the numerical tests are reported. Comparisons with experimental results are described in $\S 3$.

The main purpose of the mesh and time-step refinement studies is to determine the minimal number of mesh points and time steps necessary to obtain accurate solutions in the regions of interest. All the tests were carried out for the case of $T^{*}=6$ and $R e=2500$ using a uniform discharge velocity profile and an impulse velocity programme. The downstream boundary was placed at a distance of $40 \mathrm{~cm}$ from the orifice, while the outer boundary was at a distance of $5 \mathrm{~cm}$ away from the axis of symmetry. The focus of the present paper is on the evolution of the circulation and therefore the refinement studies are performed on this quantity.

Three meshes with $76 \times 61,151 \times 121$ and $301 \times 241$ points in the axial and radial directions, respectively, were employed in the mesh refinement study. The evolution of the total circulation for the three meshes is given in figure 3 (see $\S 3.2$ for details on the calculation of the circulation). The total circulation is not sensitive to the mesh sizes used. The circulation obtained for the medium mesh deviates less than $0.2 \%$ from that of the finest mesh. Consequently, the medium mesh of $151 \times 121$ points was used in the numerical simulations of the SDV case with uniform velocity profile. The number of mesh points for the orifice and nozzle cases is larger because of the addition of the tube region and the region outside the tube (in the nozzle case). Similarly, in the cases with farther downstream boundary, the number of mesh points was increased to maintain the same mesh density. For example, in the case of a parabolic specified discharge velocity, a mesh of $521 \times 121$ points was employed.

In the time-step-refinement study the SDV case with a mesh of $151 \times 121$ points was solved for several time steps: $\Delta t^{*}=0.06,0.03,0.015$ and 0.0075 (the respective cell-CFL numbers were $1,0.5,0.25$ and 0.125 , respectively). The evolution of the total circulation for these time steps is presented in figure 4. As the formation time $t^{*}$ 


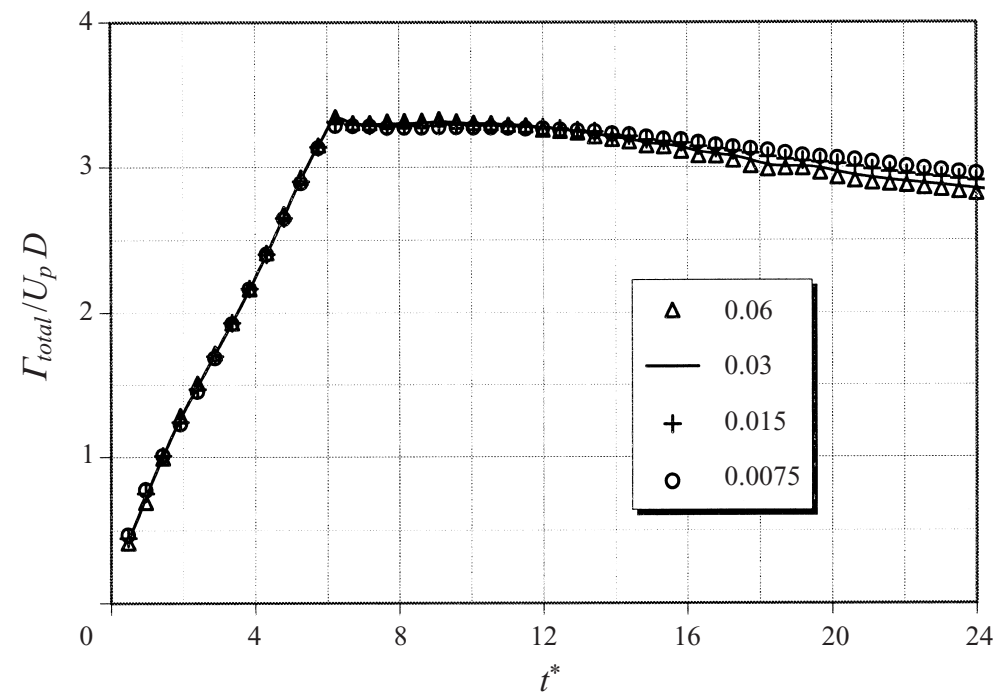

FIGURE 4. Time-step refinement study of the total circulation.
(a)
$T^{*}=2$
$(g)$
$T^{*}=6$

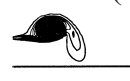

(b) (h)

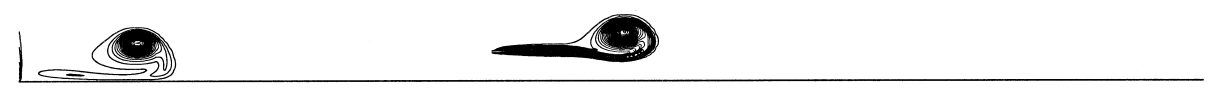

(c)

(i)

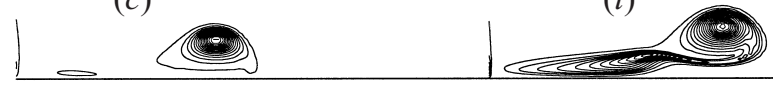

(d)

$(j)$

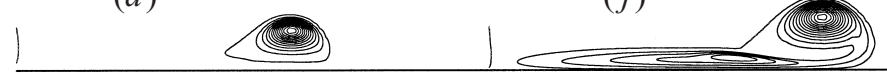

(e)

(k)

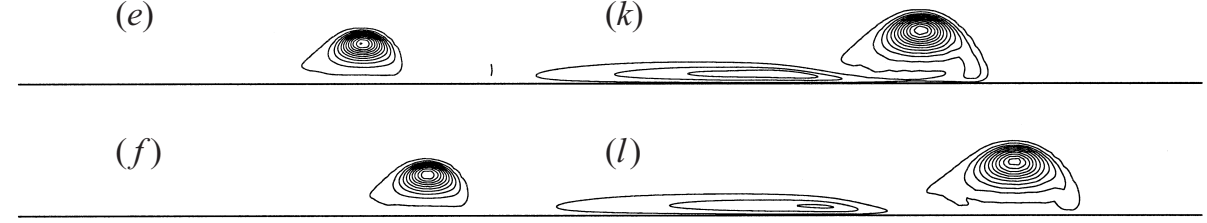

Figure 5. The formation and propagation of the vortex ring for $T^{*}=L_{m} / D=2$ and 6 . The formation times are $t^{*}=1.9(a, g), 5.8(b, h), 9.6(c, i), 13.4(d, j), 17.3(j, k)$ and $21.1(f, l)$. The non-dimensional vorticity contour lines are plotted for the range of $\omega=0.17$ to 5 with an increment of 0.33 .

increases, the magnitude of the time step has a larger effect on the solution. Plots of the vorticity field reveal that the reason is the sensitivity of the propagation velocity of the pinched-off vortex ring to the time step. Thus, it is necessary to use quite small time steps for long time simulations $\left(t^{*}>20\right)$. The present study is not concerned with the long time properties of the flow field. Rather, it focuses on $t^{*}<20$ and therefore a time step of $\Delta t^{*}=0.003$ was employed, resulting in the regions of interest less than $1 \%$ error relative to the finest time step solution. 


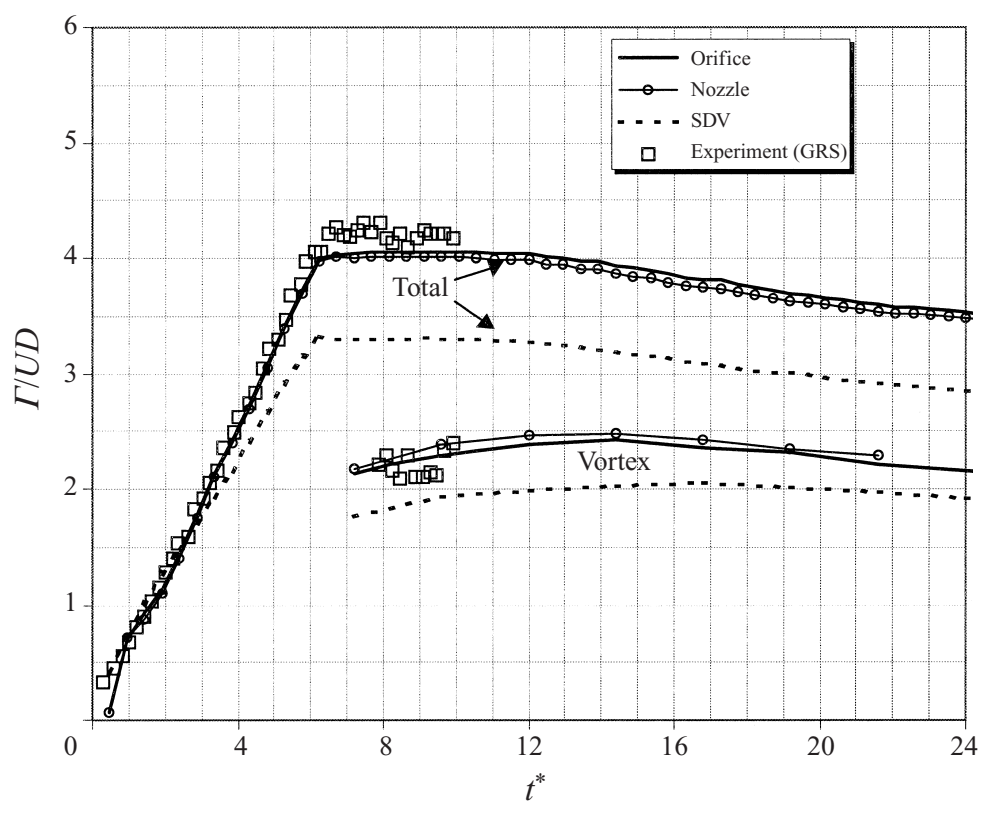

FIGURE 6. The total and vortex circulation evolution for $T^{*}=6$ and $R e=2500$.

\section{Total and vortex circulation}

\subsection{The flow for $T^{*}=2$ and 6}

The vorticity field in the formation and propagation phases of a vortex ring is shown in figure 5 for two different discharge times (piston stroke ratios) of $T^{*}=2$ and 6 (in all the cases described in the present paper $R e=2500$, unless otherwise mentioned). In the case of the short discharge time, all the vorticity generated at the orifice during the ejection is essentially entrained into the downstream propagating vortex ring, leaving behind a calm flow. The situation is very different at the higher discharge time of $T^{*}=6$ : the vortex ring eventually disconnects itself from the bulk of the flow (figure $5 k$ ), leaving behind a noticeable tail of active flow region.

Similar observations were made by GRS based on digital particle image velocimetry techniques, see their figures 4 and 5. The trail of active fluid flow observed in the experiments for the higher piston stroke ratio is stronger and significant secondary vortices develop there because the ejection time is longer $\left(T^{*}=14.5\right.$ as opposed to 6 in the present case) and the Reynolds number is more than twice as large $(R e=6000)$. Based on their experimental study, GRS concluded that there is a maximum in the circulation that a vortex ring can acquire as the maximal piston stroke increases. This maximum is reached at a piston stroke ratio of $L_{m} / D \approx 4$. If the piston stroke ratio is higher, the excess circulation accumulates in a jet-like wake as is observed in the numerical simulations as well (for $T^{*}=6$ ). Indeed, the origin of the trailing vorticity can be traced back (figure $5 i-j$ ) to the portion of the shear layer that could not be entrained into the vortex ring.

\subsection{The evolution of the total and vortex circulations}

The evolution of the total circulation and of the circulation of the vortex ring are of major interest in the present study. These quantities are plotted in figure 6 for the SDV and for the orifice cases. In all the cases to be presented, an impulse velocity 
programme is used and $T^{*}=6$, unless otherwise mentioned. The experimental results of GRS for an orifice configuration are also shown in figure 6 (the results of the nozzle case are also shown but they will be addressed only in $\S 5.2$ ). The total circulation was calculated by integrating the vorticity in the entire domain of computation. The calculation of the vortex circulation is more subjective, especially before a clear pinch-off can be observed (i.e. for $t^{*}<12$ in the present case). It was estimated by calculating the circulation inside a polygon that included the vortex ring to the best of our judgement. The circulation in figure 6 is non-dimensionalized by $U_{p} D$. In the numerical simulations, $U_{p}=30 \mathrm{~cm} \mathrm{~s}^{-1}$ and $D=2.5 \mathrm{~cm}$, while in the experiments $\bar{U}_{p} \approx 7.5 \mathrm{~cm} \mathrm{~s}^{-1}$ and $D=1.63 \mathrm{~cm}$.

In the slug flow model with a constant piston velocity $U_{p}$, the total circulation is given by $\Gamma_{\text {total }}=\frac{1}{2} U_{p}^{2} t / U_{p} D$ or in non-dimensional form $\Gamma_{\text {total }} / U_{p} D=\frac{1}{2} t^{*}$. Indeed, the computed total circulation increases almost linearly with the formation time $t^{*}$. The total circulation stops increasing after the discontinuation of the flow discharge and from then on it eventually decays slowly. The circulation of the vortex ring is almost constant after it is fully formed. The agreement between the numerical simulation of the orifice case (where the flow inside the tube is calculated as well and consequently the discharge velocity profile is not uniform) and the experimental results is very good, including the circulation of the vortex ring. The small deviation in the maximal total circulation is obtained mainly because of a slightly larger discharge time in the experiments

The calculated total circulation upon the stopping of the discharge is found for the SDV case to be $\Gamma_{\text {total }}=3.3$ whereas the slug flow model predicts $\Gamma_{\text {total }}=\frac{1}{2} T^{*}=3$. The excess circulation, as confirmed by the vorticity distribution at the orifice, was artificially generated by the boundary conditions $u_{r}=0$ imposed on the orifice. $\dagger$ It should be realized, however, that in the numerical simulation with the specified discharged velocity, the additional circulation was generated due to the imposed Dirichlet-type boundary conditions (that results in $\partial u_{r} / \partial x \neq 0$ ). In the orifice case (both in the experiments and in the numerical simulations) the total circulation is larger than that predicted by the slug flow model due to the non-uniform velocity profile.

The formation number is the formation time when the total circulation imparted by the discharging flow is equal to the circulation of the pinched-off vortex ring, GRS. Using this definition, the formation number is found to be 3.6 both in the SDV and in the orifice cases, figure 6 . This is a surprising finding since the total circulation increase rate as well as the vortex circulation of the SDV case are different from the orifice case. An identical formation number of 3.6 was obtained in the experiments.

In the calculation of the formation numbers of all these numerical and experimental cases, a vortex circulation of $\Gamma_{\text {vortex }}=2.3$ was used. This value is the mean computed for the time interval of $10>t^{*}>8$ (the same time range was used in the experiments of GRS). In the numerical simulations the evolution of the vortex circulation could be calculated for formation times up to $t^{*}=30$. The evolution of the vortex circulation only and the corresponding vorticity fields are depicted in figure 7. The vortex circulation increases up to a maximal value of 2.42 (at $t^{*}=14.4$ ). Not surprisingly, the vortex ring is not disconnected from its tail up to $t^{*} \approx 14$ and therefore the vortex circulation may increase up to that time. In this case, the maximal vortex circulation is

$\dagger$ In another simulation, we did not impose $u_{r}=0$ but used instead $\partial u_{r} / \partial z=0$, preventing the generation of vorticity at the orifice (except on the wall). In this case the slug flow value of $\Gamma_{\text {total }}=3$ was accurately reproduced. 


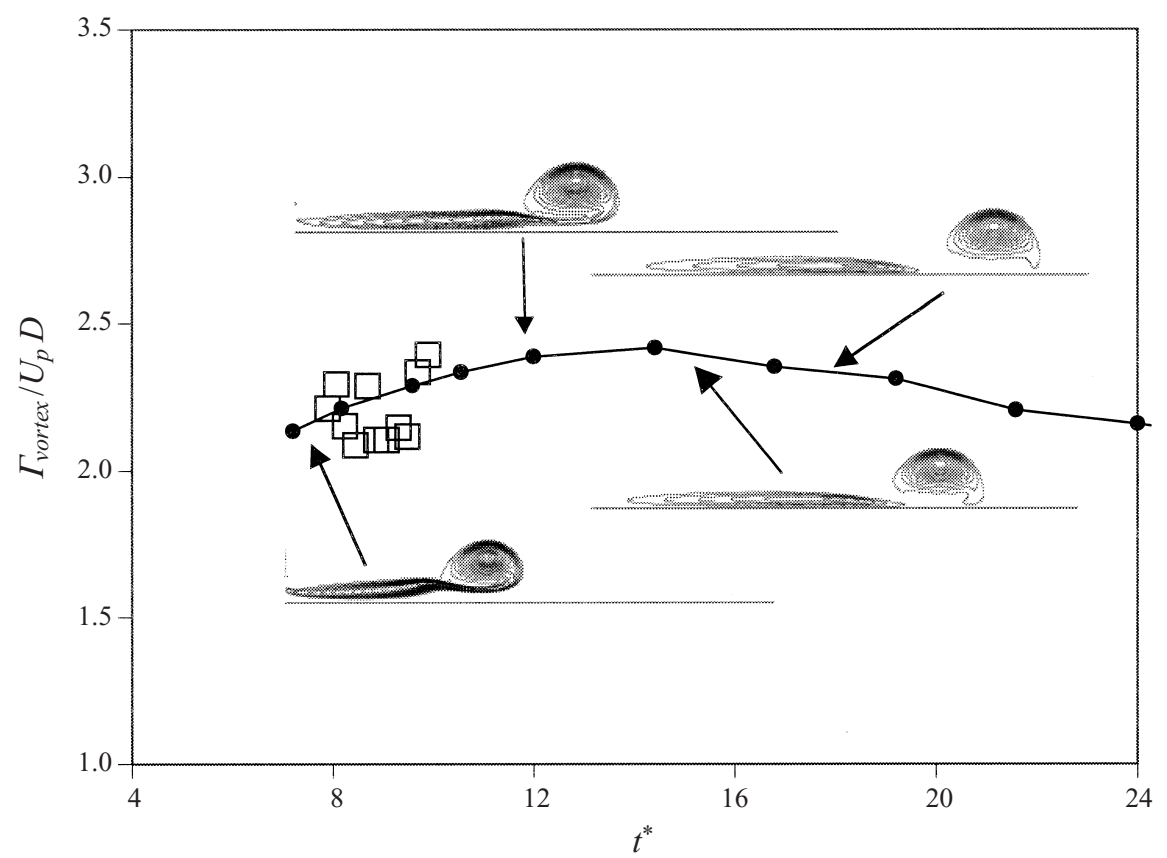

FIGURE 7. The evolution of the vortex circulation and the corresponding vorticity field for the orifice case.

obtained at the pinch-off of the vortex ring. It seems therefore reasonable to base the calculation of the formation number on the maximal value of the vortex circulation. This yields a somewhat higher formation number of 3.83 .

\subsection{Vortex circulation vs. $T^{*}$}

We discuss now the development of the flow field as a function of the discharge time $T^{*}$ (or the maximal piston stroke ratio $L_{m} / D$ ) for the SDV case. In figure 5 we pointed out the differences in the flow field for short $\left(T^{*}=2\right)$ and long $\left(T^{*}=6\right)$ discharge time. Following the experiments of GRS, we have found that the vortex ring eventually pinches off for any $T^{*}$, leaving behind a significant tail of active fluid if $T^{*}>4$. In figure 8 we present the vorticity field for the SDV case immediately after the pinch-off for several values of $T^{*}$. We define the pinch-off to occur when the contour line of $5 \%$ of the maximal vorticity in the core of the vortex encircles the vortex for the first time. Figure 8 reveals that as $T^{*}$ increases, the pinch-off occurs later and farther downstream. The maximal total circulation increases, of course, but it is obvious that for $T^{*}>4$ the excess vorticity (or circulation) is left behind in a trail that increases in length and strength with $T^{*}$. The vortex rings themselves, however, are apparently of equal size and strength for $T^{*}>4$.

An important conjecture of GRS was that for a given set of conditions, the circulation of the vortex ring attains a constant value for $L_{m} / D>4$, i.e. there is a maximum in the circulation a vortex ring can acquire during its formation as $L_{m} / D$ (or in our notation $T^{*}$ ) increases. This experimental finding, that was shown by GRS to result from the variational principal of Kelvin-Benjamin, was confirmed in the numerical simulations as well - visually in figure 8 and quantitatively in figure 9. In the latter figure, the circulation of the vortex ring is plotted vs. $T^{*}$ for an impulse velocity programme and uniform specified discharge velocity profile. The 


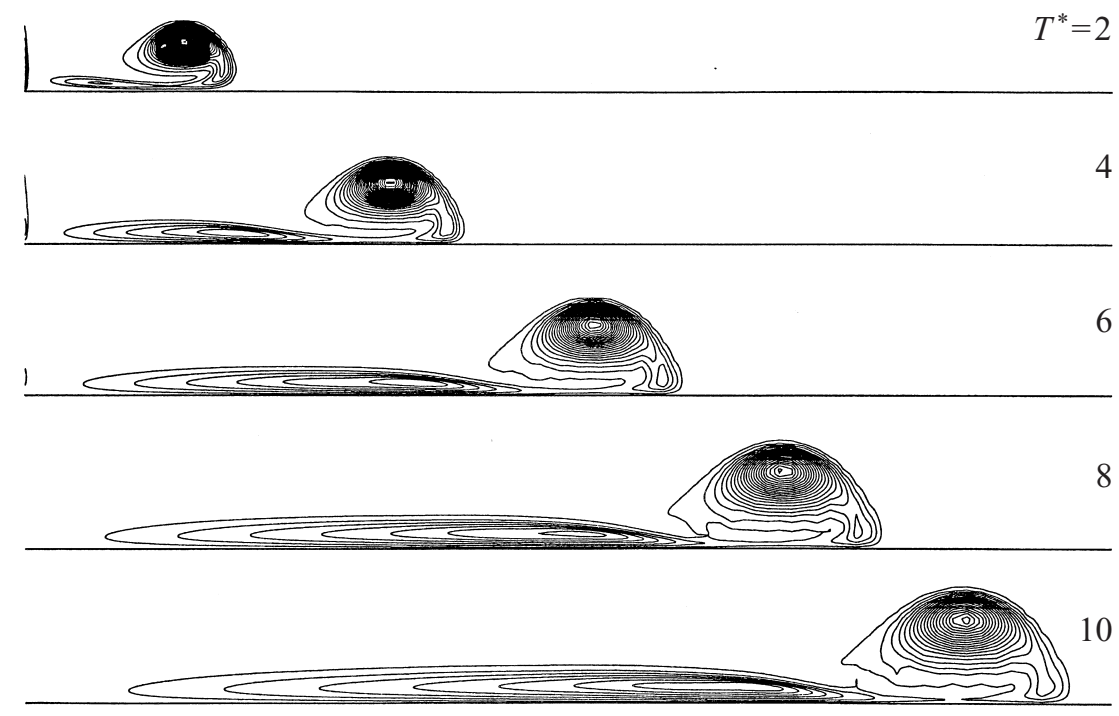

FIGURE 8 . The vorticity field close to the pinch-off of the vortex ring for several $T^{*}$.

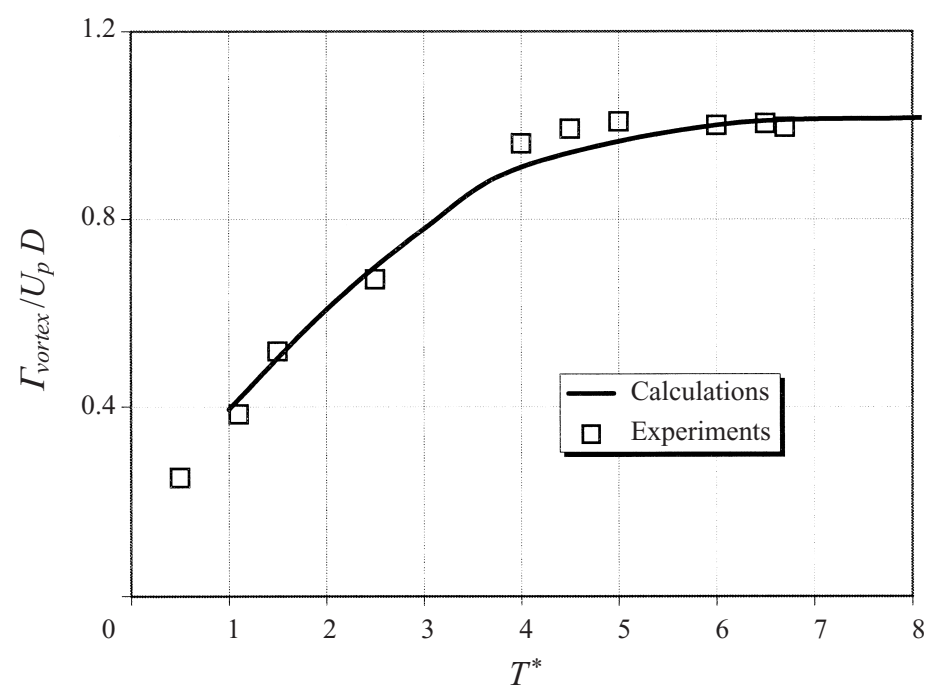

FIGURE 9. The vortex ring circulation dependence on $T^{*}\left(\right.$ or $\left.L_{m} / D\right)$.

circulation was calculated in all the cases at $t^{*} \approx 10$, similarly to the experiments. To allow meaningful comparison with the experimental results, the circulations of the numerical and experimental results were scaled to be equal at $L_{m} / D=6$. Reasonable agreement was obtained, except a deviation of approximately $8 \%$ near $T^{*}=4$.

\section{Effect of velocity programme and discharge velocity profile}

4.1. The one-dimensional vorticity flux model

Many aspects of circulation generation and vortex rings can be analysed by a onedimensional vorticity flux model. Using this approximation, the total circulation $\Gamma_{\text {total }}$ 

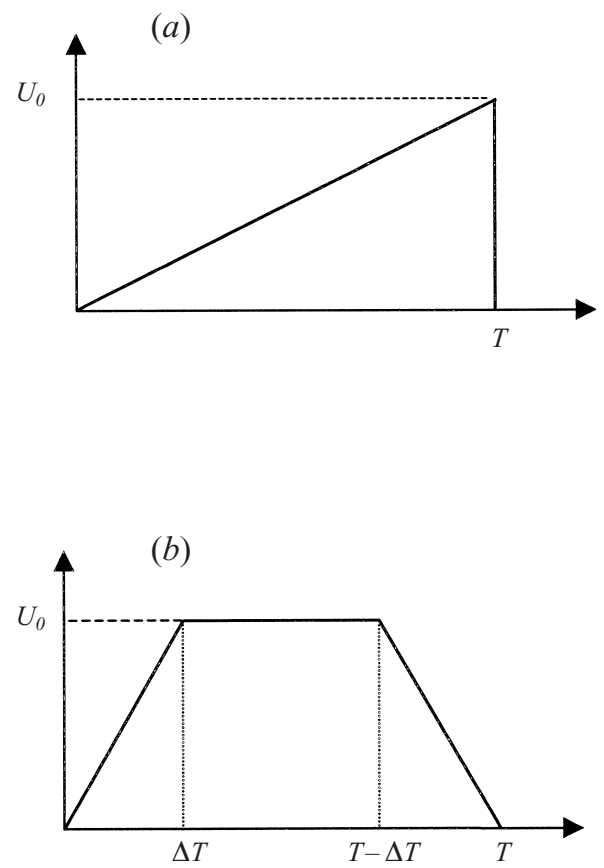

FIGURE 10. Non-impulsive velocity programmes: $(a)$ linear ramp and $(b)$ trapezoidal.

injected into the flow field can be approximated by

$$
\frac{\mathrm{d} \Gamma_{\text {total }}}{\mathrm{d} t}=\int_{0}^{R} U_{e} \omega_{e} \mathrm{~d} r=\int_{0}^{R} U_{e} \frac{\mathrm{d} U_{e}}{\mathrm{~d} r} \mathrm{~d} r=\frac{1}{2} U_{0}^{2}(t),
$$

where the subscript $e$ refers to the exit plane of the tube (the discharge plane), $R$ is the radius of the tube, $\omega$ is the vorticity and $U_{0}(t)=U_{e}(r=0, t)$ is the discharge velocity at the axis of symmetry. In external boundary layer flows $U_{0}$ can be substituted by the maximal discharge velocity (the outer velocity). In internal flows, however, (4.3) reveals that the velocity at the centre $r=0$ should be used. In the present study, the centreline velocity is also the maximal velocity, except at the very beginning of the piston motion (see figure 13). At that early time the roll-up of the vortex ring takes place and the assumption of a nearly one-dimensional flow at the exit of the tube is invalid anyway.

Thus, the total circulation imparted by the discharging flow is given by

$$
\Gamma_{\text {total }}=\frac{1}{2} \int_{0}^{t} U_{0}^{2}(\tau) \mathrm{d} \tau
$$

This relationship reveals that the total circulation depends on both the velocity programme and the velocity profile (or more accurately, under the one-dimensional approximation, on the evolution of the axial velocity profile at the centre of the orifice). In the present section, these two factors are examined.

\subsection{Effect of the velocity programme}

To study the dependence of the formation number on non-impulsive velocity programmes, linear ramp and trapezoidal velocity programmes as defined in figure 10 were employed. In all the cases the velocity programme was selected to result in the 


\begin{tabular}{llccccc}
\hline Velocity profile & Velocity programme & $\Delta T / T$ & $R e$ & $\Gamma_{\max }$ & $\Gamma_{\text {vortex }}$ & Formation number \\
Uniform & Impulse & - & 2500 & 3.33 & 2.05 & 3.60 \\
Nozzle & Impulse & - & 2500 & 3.98 & 2.47 & 3.97 \\
Orifice & Impulse & - & 1250 & 4.43 & 2.61 & 3.81 \\
Orifice & Impulse & - & 2500 & 3.99 & 2.42 & 3.83 \\
Orifice & Impulse & - & 5000 & 3.74 & 2.30 & 3.80 \\
Parabolic & Impulse & - & 2500 & 12.08 & 1.85 & 0.90 \\
Uniform & Trapezoidal & 0.1 & 2500 & 3.16 & 2.06 & 3.54 \\
Uniform & Trapezoidal & 0.2 & 2500 & 2.99 & 2.06 & 3.78 \\
Uniform & Trapezoidal & 0.3 & 2500 & 2.78 & 2.05 & 3.95 \\
Uniform & Trapezoidal & 0.4 & 2500 & 2.51 & 1.90 & 3.97 \\
Uniform & Linear & - & 2500 & 2.24 & 1.85 & 5.22
\end{tabular}

TABLE 1. Maximal circulation $\left(\Gamma_{\max }\right)$, vortex circulation $\left(\Gamma_{\text {vortex }}\right)$ and formation number dependence on generation factors for $T^{*}=6$.

same stroke ratio of $T^{*}=L_{m} / D=\int_{0}^{T} U(t) \mathrm{d} t / D=6$. This relationship also suggests the use of the instantaneous mean velocity defined by

$$
\bar{U}_{p}(t)=\frac{1}{t} \int_{0}^{t} U(\tau) \mathrm{d} \tau
$$

as the scaling velocity for the calculation of the formation time of non-impulsive velocity programmes. Most previous works, with the exception of GRS and Johari et al. (1996), do not use (4.5) as the scaling velocity. Rather, they prefer to define a virtual origin in time to account for non-impulsive velocity programmes (e.g. Didden 1979; James \& Madnia 1996).

The symmetric trapezoidal velocity programme in the present numerical simulations is defined by a single parameter: $\Delta T / T$. Four cases with $\Delta T / T=0.1,0.2,0.3$ and 0.4 were simulated for the specified uniform discharge velocity case. The formation numbers of these cases as well as a linear ramp case $(\Delta T / T=1)$ and the impulse case $(\Delta T / T=0)$ are given in table 1 (together with other cases and parameters not discussed in the present section). In all these cases, the maximal vortex circulation was used in the calculation of the formation number. The formation number increases only slightly with the increase in $\Delta T / T$, i.e. as the accelerating and decelerating phases last a larger part of the total discharge time. Yet, even for $\Delta T / T=0.4$, the increase in the formation number is only $10 \%$ relative to the impulse velocity programme. In the case of uniform acceleration (linear ramp), however, the formation number increases to $F=5.22$, still within the experimental bounds of $5.5>F>4.5$ established by Johari et al. (1996) for linear ramp programmes.

\subsection{Effect of discharge velocity profile}

Another issue is the dependence of the formation number on the discharge velocity profile. In the specified uniform velocity profile case, the discharged jet produces a very thin shear layer near the wall. The other limiting case is the Poiseuille velocity profile, where the vorticity is distributed along the entire orifice. In real flow cases, the velocity profile is close to uniform at the starting of the piston motion (with a peak near the wall, Didden 1979), and it approaches a parabolic velocity profile as the piston is pushed forward. In the present work, the capability of the numerical simulations to impose these two limits separately was utilized. Contrary to the experiments, in the numerical simulations the discharge velocity profile (either uniform or parabolic) 
(a)

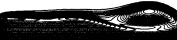

(b)

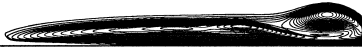

(c)

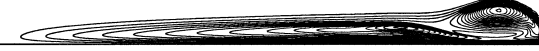

(d)

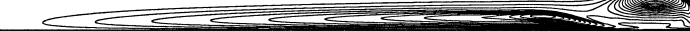

(e)

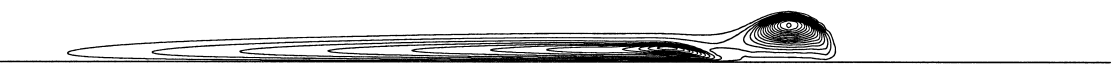

(f)

FiguRE 11. The vorticity field for the parabolic discharge velocity $\left(T^{*}=6\right.$ and $\left.R e=2500\right)$. The formation times are $(a) t^{*}=4.3,(b) t^{*}=7.2,(c) t^{*}=9.6,(d) t^{*}=14.4,(e) t^{*}=19.2$ and $(f)$ $t^{*}=24.0$. The non-dimensional vorticity contour lines are plotted for the range of $\omega=0.17$ to 17 with an increment of 0.83 .

could be maintained constant over the whole duration of the velocity programme. This allows the effect of each limiting velocity profile on the flow field in general and on the total and vortex circulations in particular to be isolated.

The results for the uniform discharge velocity profile were elaborated in $\S 3$ and in $\S \S 4.1$ and 4.2. In the present subsection, we discuss the flow resulting from a specified parabolic discharge velocity profile of $U=U_{0}\left(1-r^{2} / R^{2}\right)$, using an impulse velocity programme and a discharge time of $T^{*}=6$. The average velocity was maintained identical to the uniform discharge velocity case and consequently $U_{0} / U_{p}=2$. Figure 11 presents a time sequence of the vorticity field for this case. The most obvious difference with the equivalent uniform velocity case (figure 5) is the existence of a thick shear layer of $O(1)$. Although a vortex ring is formed in this case as well, it has distinctive geometric characteristics. The vortex centre is closer to the axis of symmetry and its shape is more slender because of the larger axial velocity near the axis of symmetry.

The evolution of the total and vortex circulations is shown in figure 12. Equation (4.4) predicts that the total circulation increase rate is four times larger than that of the uniform velocity case (since $U_{0} / U_{p}=2$ ). Indeed, the maximal total circulation at $t^{*}=T^{*}=6$ is four times as large. In this case as well, the excess vorticity is not entrained into the vortex ring but it rather accumulates in a tail that is significantly longer and stronger than in the uniform velocity profile. Its downstream edge is strong enough to form a secondary vortex ring. The concentration of large vorticity in the tail near the axis of symmetry increases the destruction of vorticity (and total circulation) by the vorticity cancellation mechanism. The decay of the vortex vorticity, however, is significantly weaker because it is away from the axis of symmetry.

In the case of a parabolic profile the vortex ring disconnects itself later and therefore vortex circulation could be calculated only for $t^{*}>16$. In spite of the significantly 


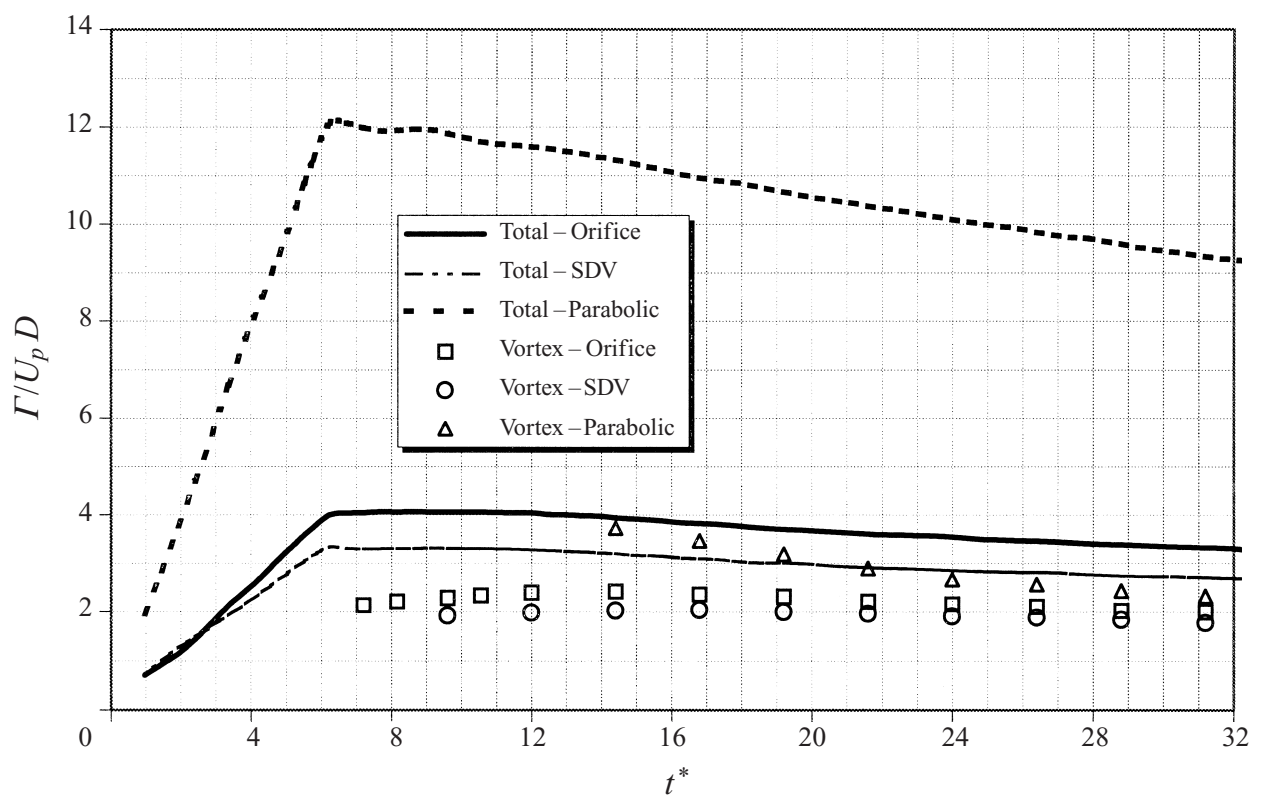

FiguRE 12. The evolution of the total and vortex circulations for the uniform and parabolic discharge velocity and the orifice cases $\left(T^{*}=6\right.$ and $\left.R e=2500\right)$.

larger total circulation, the calculated circulation of the vortex ring is $\Gamma_{\text {vortex }}=1.85$, close to the value obtained for the uniform discharge velocity case $\Gamma_{\text {vortex }}=2.05$. The theoretical value of the vortex circulation (based on the variational principle of Kelvin-Benjamin applied to the parabolic discharge velocity) is 1.65 , K. Shariff (1997, personal communication), in reasonable agreement with the calculated value.

The formation number for the parabolic velocity distribution is found to be $F=0.91$, one quarter of the formation number obtained for the uniform velocity profile. This is apparently a significant deviation from the universal formation number of $F \approx 4$ found in the experiments and in the uniform profile numerical simulations. It is, however, in good agreement with the theoretical prediction of GRS based on the Kelvin-Benjamin variational principle. This latter analysis results in $F=0.87$ (assuming the limiting non-dimensional kinetic energy of the vortex ring is equal to that obtained for the uniform velocity case).

As mentioned, in realistic cases the velocity profile of the discharged flow is neither uniform nor parabolic; rather it develops with time. Figure 13(b) presents the calculated evolution of the axial discharge velocity for the orifice case and $R e=2500$ (figure 13 also shows the discharge velocity for $R e=1250$ and 5000; these two cases will be discussed in $\S 5.1$ ). At the very start of the flow discharge, the velocity is nearly uniform with a small peak near the wall. This peak leads to a centreline velocity smaller than the average velocity. However, this peak decreases rapidly with time and for $t^{*}>1$ the centreline velocity is larger than the average velocity. Therefore, the total circulation increase rate is larger than in the SDV case. As time passes, the centreline velocity and the width of the boundary layer increase and the velocity approaches a parabolic profile. However, for a stroke ratio of $T^{*}=6$ the velocity profile is still far from being parabolic.

The evolution of the total and vortex circulations for the orifice case are also shown in figure 12. The total circulation curve of the orifice case lies in between the two 

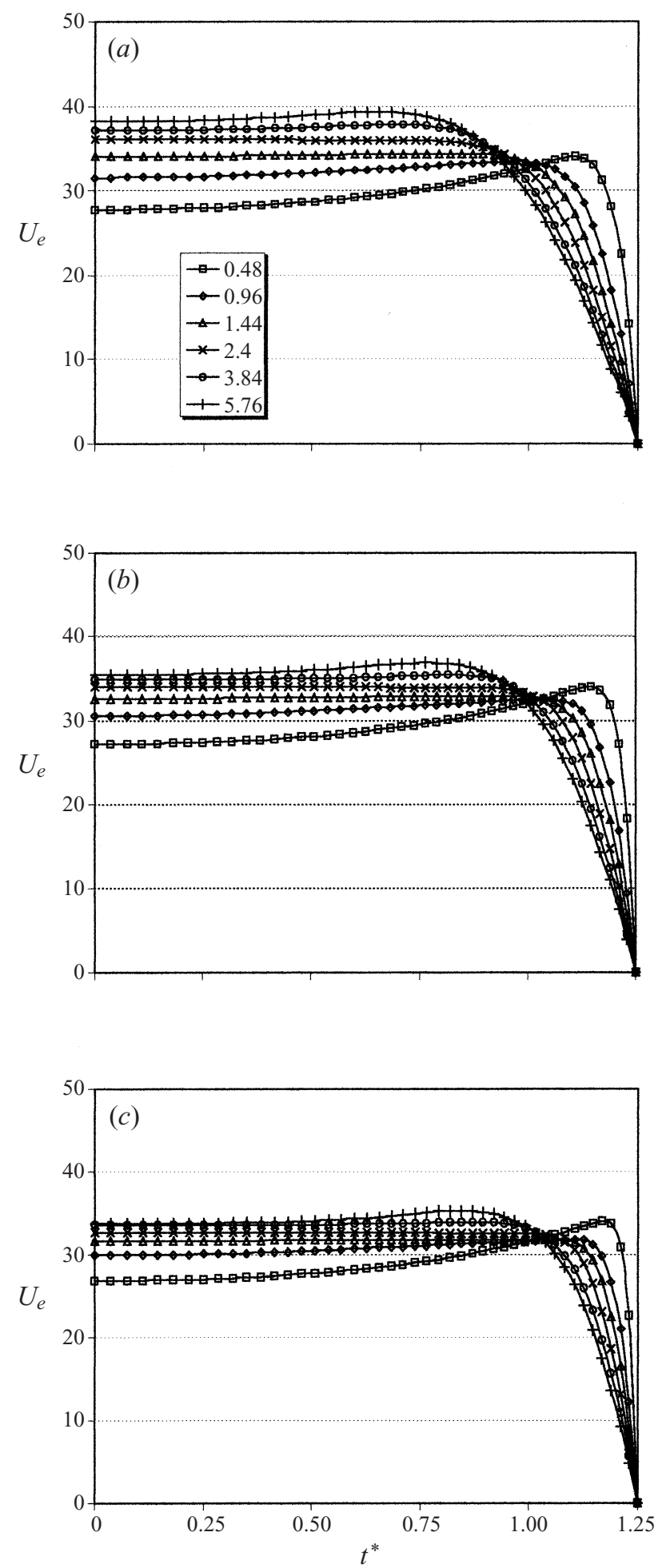

FIGURE 13. The evolution of the discharge velocity for the orifice case. (a) $R e=1250$ (b) $R e=2500$, (c) $R e=5000$. The various lines refer to the formation time (see legend in $a$ ). 
limiting cases of uniform and parabolic discharge velocity. It is closer, however, to the uniform velocity case, as might have been expected because the discharge velocity profile in the orifice case is closer in shape to a uniform profile than to a parabolic one (figure 13b). Similar trends are found in the vortex circulation, although the differences in the magnitudes are considerably smaller.

An important implication of these findings is that variations in the velocity profile affect the evolution of the total circulation and therefore the formation number as well. Factors such as the velocity program, $L_{m} / D, R e$ and the configuration geometry affect the instantaneous velocity profile of the discharged jet (see also $\S 5.1$ and 5.2). In most common cases, the variations are usually small (e.g. the piston stroke ratio is relatively small $L_{m} / D<10$ ), and therefore the range of formation numbers found in the experiments of GRS is narrow. Thus, the formation number depends weakly on the velocity programme but strongly on the velocity profile of the discharged flow. It does not depend, however, on the discharge time (or piston stroke ratio) beyond a threshold value of $T^{*} \approx 4$.

To explain these findings, table 1 lists the maximal total circulation and the vortex circulation for $T^{*}=6$ and various velocity programs (impulse, trapezoidal or linear ramp), velocity profiles (uniform or parabolic), $R e$ and configuration geometry. In all the wide variety of cases considered in table 1 , the vortex circulation (scaled by $U_{p} D$, where $U_{p}$ is the maximal piston velocity) is in the range of $2.61>\Gamma_{\text {vortex }}>1.85$. The maximal circulation for the same cases varies in a much wider range of $12.08>\Gamma_{\max }>$ 2.24. Theoretical as well as experimental results also indicate that the variations in $\Gamma_{\text {vortex }}$ are indeed relatively small. An analysis based on the variational principle of Kelvin-Benjamin (assuming a constant limiting value of the non-dimensional kinetic energy) predicts $1.90>\Gamma_{\text {vortex }}>1.42$ for cases with specified discharge velocity profile, while the experimental results of GRS are in the range of $2.7>\Gamma_{\text {vortex }}>2.2$.

These observations might hint at the possibility that the vortex circulation (scaled properly) is yet another universal quantity related to vortex rings. In other words, the vortex circulation is relatively insensitive to the formation conditions, once its asymptotic state (e.g. $T^{*}>4$ ) has been reached. This conjecture should be further investigated in the future.

The total circulation, however, depends strongly on both the velocity program and velocity profile. Equation (4.4) reveals that the total circulation should indeed depend both on the velocity profile and velocity programme. Consequently, the formation number might vary significantly since it is determined by the intersection of $\Gamma_{\text {vortex }}$ (that weakly depends on the velocity programme and profile) with the curve $\Gamma_{\text {total }}(t)$ (that strongly depends on the velocity programme and velocity profile). Therefore, the formation number for each of the cases solved (also listed in table 1) varies in a wider range of $5.2>F>0.9$. It increases for velocity programs with gradual growth rate of the velocity (because the slope of the total circulation decreases) and decreases significantly for the parabolic velocity profile (the slope increases because $U_{0}>U_{p}$ ).

\section{Other factors affecting the formation number}

\subsection{Reynolds number}

Most experiments considered turbulent flows. Several studies indicated that the Reynolds number (within a certain range) played an insignificant role in the evolution of the circulation of laminar vortex rings (GRS; Didden 1979). Other works, Maxworthy (1977) or James \& Madnia (1996), found that Re did play a role through the 

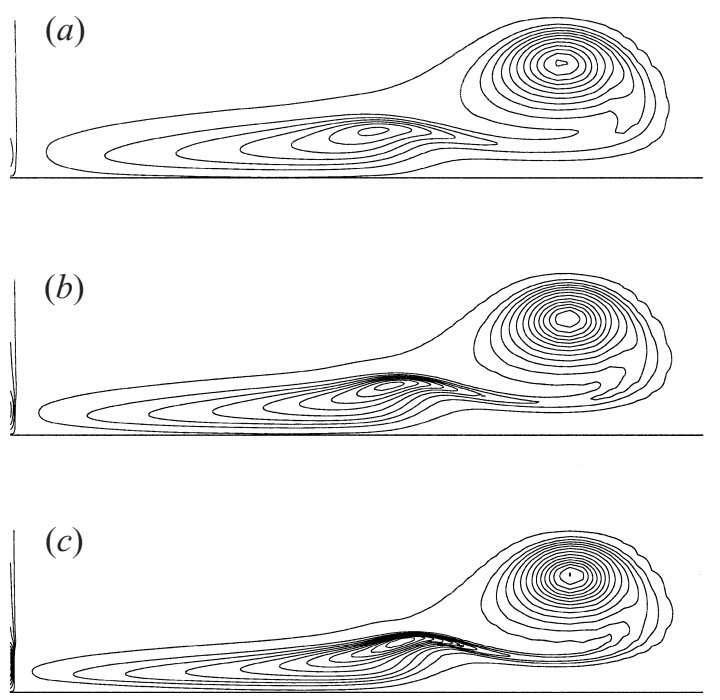

FIgURE 14. The effect of $R e$ on the vorticity field at $t^{*}=10\left(T^{*}=6\right)$. The non-dimensional vorticity contour lines are plotted for the range of $\omega=0.17$ to 5 with an increment of 0.33 . (a) $R e=1250$, (b) $R e=2500,(c) R e=5000$.

modification of the discharge velocity profile. To test the dependence of the formation number and the evolution of circulation on the Reynolds number, the case of $T^{*}=6$ with impulse velocity programme was simulated for $R e=1250$ and 5000, in addition to the base case of $R e=2500$. These $R e$-dependence tests have been made for both the SDV and the orifice cases. The former case of specified discharge velocity tests only the effect of $R e$ on the roll-up of the vortices and their propagation, while the orifice case adds in the effect of variations in the discharge velocity profile.

The vorticity field for these three Reynolds numbers at $t^{*}=10$ is shown in figure 14 for the SDV case. As expected, the vorticity gradients increase as the Reynolds number increases, both in the vortex ring region and in the tail region. Particularly noticeable is the thinning of the feeding shear layer with the increase in $R e$. The evolution of the circulation of these cases is shown in figure 15. The total circulation imparted to the flow is $R e$-independent in the SDV case, figure 15(a), because the discharge velocity profile is pre-specified (i.e. it is independent of $R e$ ). The total circulation is also independent of the Reynolds number for a short formation time after the stopping of the discharge. However, for $t^{*}>10$ the decay of the total circulation increases with the decrease in Re. James \& Madnia (1996) attributed it to vorticity cancellation at the axis of symmetry. Indeed, an examination of the vorticity field indicates that the total circulation is constant as long as the tail of the vortex ring is away from the axis of symmetry, see figures 5 and 6 . As soon as the tail reaches the vicinity of the axis of symmetry (figure 5i), significant vorticity cancellation is observed and the total circulation decays at an increased rate. The circulation of the vortex ring itself is $R e$-independent for $14>t^{*}>10$. At later times the vortex circulation does depend on $R e$, but the decay rate is significantly less than that of the total circulation, as was also observed by James \& Madnia (1996). The reason is the weaker vorticity cancellation of the vortex ring due to its distance from the axis of symmetry. The effect of Re on the formation number is negligible in the SDV case.

If the discharge velocity profile does depend on $R e$, such as in the orifice case, the evolution of the circulation is $R e$ dependent as figure $15(b)$ reveals. The total 

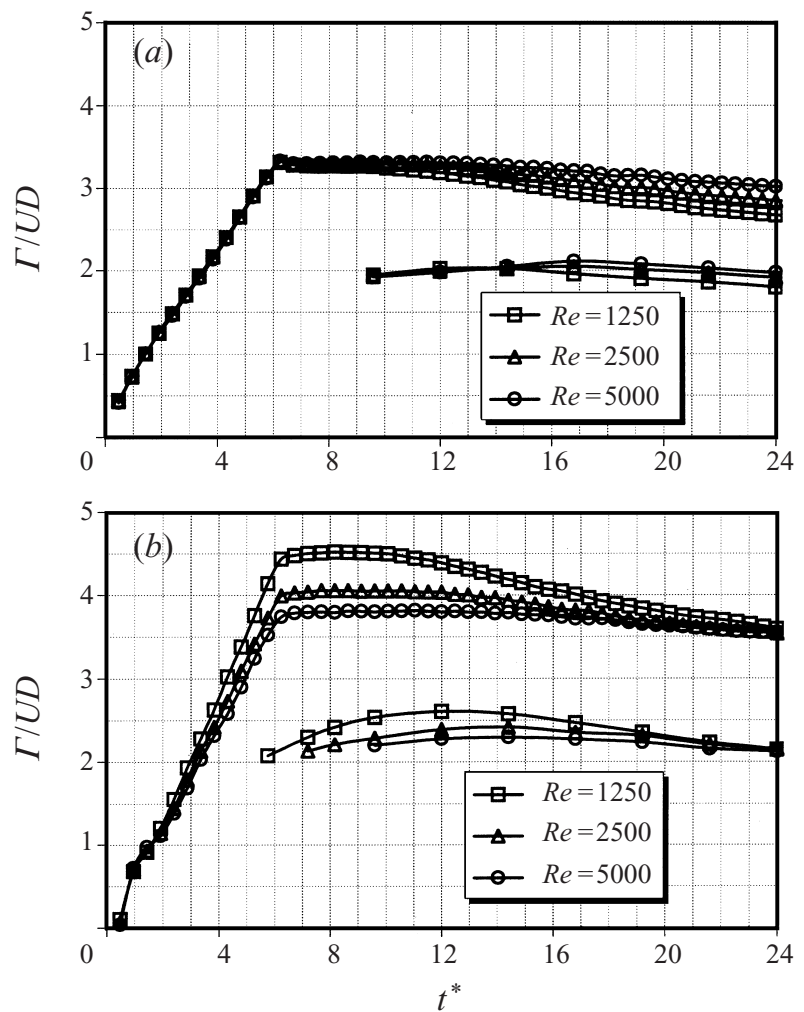

FIGURE 15. The effect of Re on the evolution of the total and vortex circulations for $(a)$ SDV and (b) orifice cases.

circulation increase rate and consequently the maximal circulation (at $t^{*}=6$ ) are larger for smaller Re. Similarly to the SDV case, the total circulation is constant after the stopping of the discharge of fluid up to $t^{*}=12$. From then on, the total circulation decays with an increased rate as $R e$ decreases, very similarly to that observed for the SDV case. In the orifice case, there are also larger changes in the vortex circulation. Similarly to the evolution of the total circulation, the vortex circulation increases with the decrease of Re. However, the formation number (based on the maximal vortex circulation) is still insensitive to $R e: 3.82 \pm 0.01$.

The increase in the total circulation as the Reynolds number decreases is a result of the dependence of the discharge velocity profile on $R e$ as shown in figure 13. Figure 13 clearly demonstrates that in the low-Re cases, the centreline velocity is larger because the boundary layer is thicker due to the higher viscosity. The larger centreline velocity increases the total circulation, as is predicted by the one-dimensional model (4.4).

Didden (1979) found experimentally that $\Gamma_{\max } / \Gamma_{0}>1$, where $\Gamma_{0}$ is the total circulation as predicted by the slug flow model. All our calculations, as well as the experimental results of GRS result in a similar relationship. In contrast, Maxworthy (1977) found that $\Gamma_{\max } / \Gamma_{0} \approx 1$. The experiments of Didden and GRS as well as our numerical simulations considered laminar flow while Maxworthy's experiments were in the turbulent regime. We interpret the disagreement to be a consequence of the different velocity profile of the discharged jet. In the turbulent case the velocity profile is approximately uniform $\left(U_{0} / U_{p} \approx 1\right.$ and therefore $\left.\Gamma_{\max } / \Gamma_{0} \approx 1\right)$ while in the laminar case the velocity profile is non-uniform $\left(U_{0} / U_{p}>1\right.$ and therefore $\left.\Gamma_{\max } / \Gamma_{0}>1\right)$. 

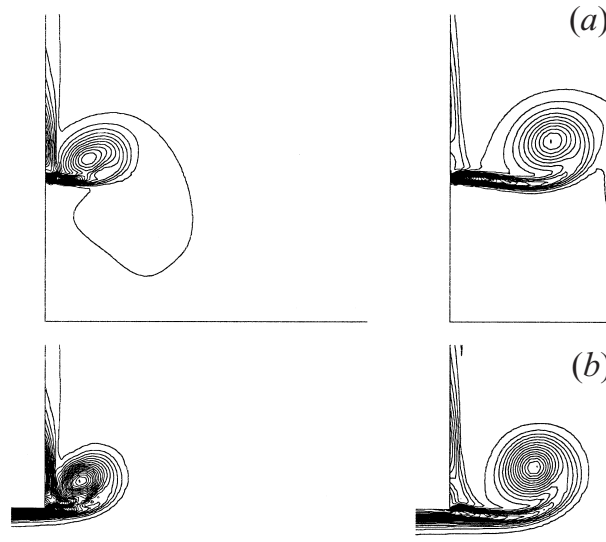

(c)
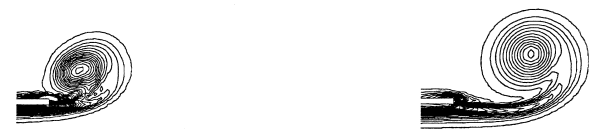

(a)

(b)
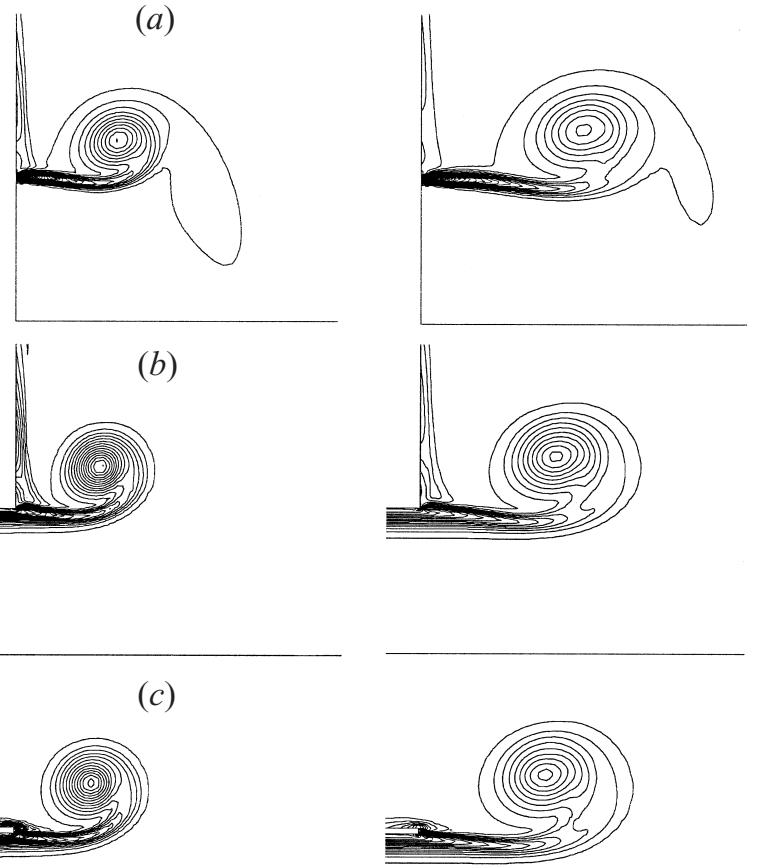

FIGURE 16. The early formation of the vortex ring for $(a)$ the SDV, $(b)$ orifice and $(c)$ nozzle cases. The formation times are $t^{*}=0.6$ (left column), $t^{*}=1.2$ (middle column) and $t^{*}=1.8$ (right column).

\subsection{Generator configuration}

Different geometrical set-ups were used in the various experimental studies to generate vortex rings. The most popular was the nozzle case (Maxworthy 1977; Didden 1979; GRS) but orifices were used as well (Maxworthy 1977; Baird, Wairegi \& Loo 1977; Glezer 1988; GRS). In the present work, the orifice and nozzle cases were solved in addition to the SDV case to study the effect of the generator configuration. Some of these results have already been discussed in the present paper.

The early formation of the vortex ring is shown in figure 16 for the three cases of (i) specified discharge velocity, (ii) orifice and (iii) nozzle cases. Two major differences can be noticed. First, the vorticity magnitude as well as the vorticity gradient are smaller for the SDV case, while for the orifice and nozzle cases, the differences in the vorticity distribution are negligible. Moreover, in the SDV case, the feeding shear layer is thinner because a uniform velocity is specified, while in the other two cases a boundary layer is developed inside the tube. Secondly, the absence of a sidewall in the nozzle case allows the roll-up of the vortex ring very close to the discharge outlet. Consequently, the axial position of the vortex ring centre is nearer in the latter case. However, this difference disappears farther downstream.

Vorticity cancellation can be found at the very early stages due to the presence of the sidewalls (the orifice case) or the outer part of the tube (the nozzle case). However, this does not induce significant differences either in the vorticity field between the orifice and the nozzle cases or in the evolution of the total circulation, see figure 6 . The total circulation of the nozzle case shows consistently lower values, but by a very 
small margin of less than $2 \%$. In the nozzle case, entrainment from the outer side of the nozzle makes the discharge velocity profile flatter and consequently the centreline velocity is smaller than in the orifice case (but by a very small amount). James \& Madnia (1996) came to similar conclusions (for $L_{m} / D<4$ ). They found that the total circulation is insensitive to the geometry of the vortex generator (nozzle or orifice) during the later time of the stroke, when the discharge velocity profiles are similar in both cases.

The difference in the vortex circulation, however, is larger by as much as $5 \%$. The lower vortex circulation of the orifice case is probably a result of enhanced vorticity cancellation of the vortex ring during its initial formation by the presence of the sidewall. This leads to a formation number of 3.83 and 3.97 for the orifice and nozzle cases, respectively (based on the maximal value of the vortex circulation), a relative difference of less than $4 \%$. In the experiments of GRS a similar trend was found, but the difference in the formation number was larger (3.6 and 4.2 for the orifice and nozzle cases, respectively).

\section{Concluding remarks}

The present numerical simulations of the formation of laminar vortex rings confirm the experimental and analytical findings of Gharib et al. (1998) and extend them to additional cases, including cases with thick shear layers. For thin shear layers, the formation number is confirmed to be approximately equal to four for a wide range of cases differing in the discharge time (piston stroke ratio), velocity programme, generator configuration and Reynolds number.

The finding of Gharib et al. (1998) of the existence of a maximum in the circulation a vortex ring can acquire as the stroke ratio increases was also confirmed. A new finding of the present study is that the maximal vortex circulation (scaled by the maximal discharge velocity and the diameter of the orifice) is only weakly dependent on the discharge velocity profile or velocity programme. In contrast, the total circulation does depend on the velocity profile (it is approximately proportional to $\bar{U}_{0}^{2}$ ) and to some extent on the velocity programme. Consequently, the formation number (that is determined by the intersection of the vortex circulation with the total circulation curve) depends on the discharge velocity profile and on the velocity programme. Non-uniform velocity profiles tend to decrease the formation number, while non-impulsive velocity programmes increase the formation number. The numerical simulations suggest that the experimentally observed variations in the formation number are principally attributable to the different evolution of the discharged velocity profile.

The relative change in the formation number was found to be as large as $400 \%$ (e.g. between the specified uniform and parabolic discharge velocity cases). The corresponding relative change of the scaled vortex circulation was found to vary less than $40 \%$. This might hint at the possibility that the scaled vortex circulation is an invariant (or at least a relatively insensitive) variable of steady vortex rings. Support for this conjecture can be found in the theoretical and experimental results of GRS and Shariff (1997, personal communication).

In all these cases, the vortex circulation is scaled by $U_{p} D$, where $U_{p}$ is the maximal piston velocity. However, this velocity scale is probably not the best choice for nonuniform or non-impulsive discharge velocity profiles. It is possible that other scaling velocities can be defined such that an even smaller variation will be obtained in the scaled vortex circulation. 


\section{REFERENCES}

Baird, M. H. I., WaIREgI, T. \& LoO, H. J. 1977 Velocity and momentum of vortex rings in relation to formation parameters. Can. J. Chem. Engng 55, 19-26.

DidDEN, N. 1979 On the formation of vortex rings: Rolling-up and production of circulation. Z. Angew. Mech. Phys. 30, 101-116.

Gharib, M., RAmbod, E. \& Shariff, K. 1998 A universal time scale for vortex ring formation. J. Fluid Mech. 360, 121-140 (referred to herein as GRS).

Glezer, A. 1988 The formation of vortex rings. Phys. Fluids 31, 3532-3541.

James, S. \& Madnia, C. K. 1996 Direct numerical simulation of a laminar vortex ring. Phys. Fluid 8, 2400-2414.

Johari, H., Dabiri, D., Weigand, A. \& Gharib, M. 1996 On the relationship between the formation number and passive scalar pinch-off in starting jets. Proc. 8th Intl Symp. Applications of Laser Techniques to Fluid Mechanics, Lisbon, Portugal, July 1996 (ed. R. J. Adrian, D. F. G. Durao, F. Durst, M. V. Heitor, M. Maeda \& J. H. Whitelaw). Springer.

LiM, T. T. \& Nickels, T. B. 1995 Vortex rings. In Fluid Vortices (ed. S. I. Green), pp. 95-153. Kluwer. Maxworthy, T. 1977 Some experimental studies of vortex rings. J. Fluid Mech. 81, 465-495.

Nitsche, M. \& Kransy, R. 1994 A numerical study of vortex ring formation at the edge of a circular tube. J. Fluid Mech. 276, 139-161.

Orlandi, P. \& Verzicco, R. 1993 Vortex rings impinging on walls: axisymmetric and threedimensional simulations. J. Fluid Mech. 256, 615-645.

Saffman, P. G. 1992 Vortex Dynamics. Cambridge University Press.

Shariff, K. \& LeOnard, A. 1992 Vortex rings. Ann. Rev. Fluid Mech. 24, 235-279.

Stanaway, S. K., Cantwell, B. J. \& Spalart, P. R. 1988 Navier-Stokes simulations of axisymmetric vortex rings. AIAA Paper 88-0318.

Weidman, D. P. \& Riley, N. 1993 Vortex ring pairs: numerical simulation and experiment. J. Fluid Mech. 257, 311-337. 